\title{
Traumatic and spontaneous intracranial hemorrhage in atrial fibrillation patients on warfarin
}

Heidi Lehtola, MD*, Antti Palomäki, MD, PhD*, Pirjo Mustonen, MD, PhD, Päivi Hartikainen, MD, PhD, Tuomas Kiviniemi, MD, PhD, Henri Sallinen, BM, Ilpo Nuotio, MD, PhD, Antti Ylitalo, MD, PhD, K.E. Juhani Airaksinen, MD, PhD*, and Juha Hartikainen, MD, PhD*

Neurology: Clinical Practice August 2018 vol. 8 no. 4 311-317 doi:10.1212/CPJ.0000000000000491
Correspondence

Dr. Lehtola

heidi.m.lehtola@gmail.com

\section{Abstract}

\section{Background}

Intracranial hemorrhage is the most devastating complication in patients with atrial fibrillation $(\mathrm{AF})$ receiving oral anticoagulation (OAC). It can be either spontaneous or caused by head trauma. We sought to address the prevalence, clinical characteristics, and prognosis of traumatic and spontaneous intracranial hemorrhages in AF patients on OAC.

\section{Methods}

Multicenter FibStroke registry of 5,629 patients identified 592 intracranial hemorrhages during warfarin treatment between 2003 and 2012.

\section{Results}

A large proportion (40\%) of intracranial hemorrhages were traumatic. Of these, $64 \%$ were subdural hemorrhages (SDHs) and $20 \%$ intracerebral hemorrhages (ICHs). With respect to the spontaneous hemorrhages, $25 \%$ were SDHs and $67 \%$ ICHs. Patients with traumatic hemorrhage were older ( 81 vs 78 years, $p=0.01)$ and more often had

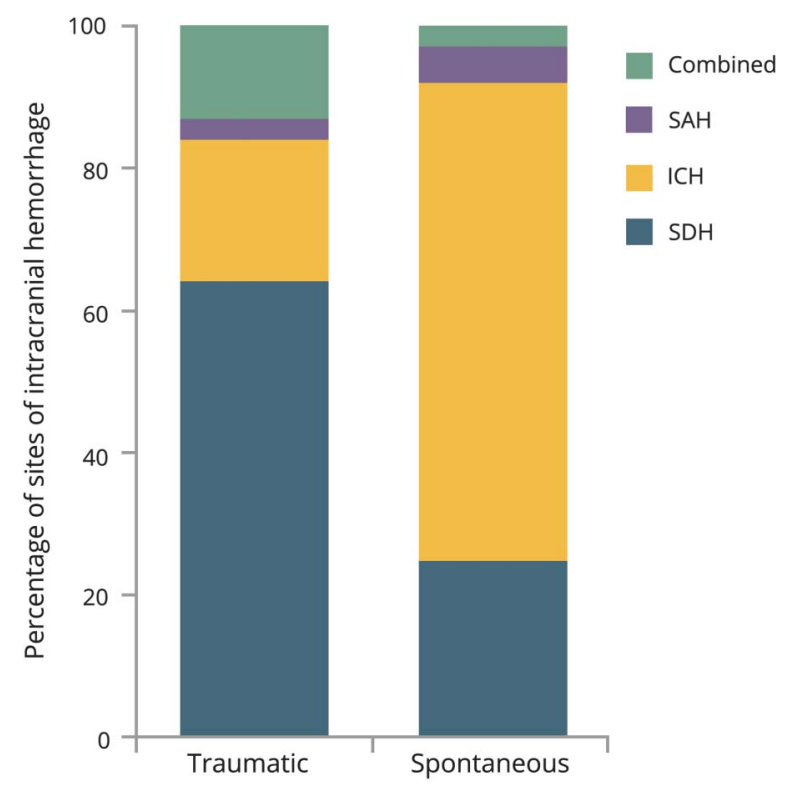
congestive heart failure ( $30 \%$ vs $16 \%, p<0.01)$ and anemia ( $7 \%$ vs $3 \%, p=0.03)$ compared to patients with spontaneous hemorrhage. Admission international normalized ratio (INR) values (2.7 vs 2.7, $p=0.79$ ), as well as $\mathrm{CHA}_{2} \mathrm{DS}_{2}$-VASc (median 4 vs $4, p=0.08$ ) and HAS-BLED (median 2 vs $2, p=0.05)$ scores, were similar between the groups. The 30-day mortality after traumatic hemorrhage was significantly lower than after spontaneous hemorrhage $(25 \%$ vs $36 \%, p<0.01)$.

\section{Conclusions}

A significant proportion of intracranial hemorrhages in anticoagulated $\mathrm{AF}$ patients were traumatic. Traumatic hemorrhages were predominantly SDHs and less often fatal when compared to spontaneous hemorrhages, which were mainly ICHs. Admission INR values as well as $\mathrm{CHA}_{2} \mathrm{DS}_{2}$-VASc and HAS-BLED scores were similar in patients with spontaneous and traumatic intracranial hemorrhage.

\section{Clinicaltrials.gov identifier \\ NCT02146040.}

\footnotetext{
*These authors contributed equally to this work.

Department of Medicine (HL, PM), Keski-Suomi Central Hospital, Jyvaskyla; Department of Cardiology (HL), Oulu University Hospital; Heart Center (AP, TK, HS, AY, KEJA) and Department of Acute Internal Medicine (IN), Turku University Hospital; NeuroCenter (PH), Kuopio University Hospital; University of Turku (IN, KEJA); Satakunta Central Hospital (AY), Pori; and Heart Center $(\mathrm{JH})$, Kuopio University Hospital and University of Eastern Finland.

Funding information and disclosures are provided at the end of the article. Full disclosure form information provided by the authors is available with the full text of this article at Neurology.org/cp.
} 
Intracranial hemorrhage is the most feared complication of oral anticoagulation (OAC) and carries a high risk for permanent disability and high mortality. The prevalence of intracranial hemorrhage in patients with atrial fibrillation $(\mathrm{AF})$ and warfarin treatment has been reported to be $0.37 \%-0.85 \%$ per year. ${ }^{1-5}$ Previously, it has been reported that the majority (73\%-93\%) of intracranial hemorrhages in patients on OAC are spontaneous $^{2,4}$ and despite adequate anticoagulation reversal, mortality has remained high. ${ }^{6}$ Old age, frailty, comorbidities, and polypharmacy increase the risk of falls and traumatic bleeding. ${ }^{7-12}$ The fear of an intracranial hemorrhage is a common reason for withdrawal of OAC in AF patients, especially in the elderly population. On the other hand, elderly patients with comorbidities also are susceptible to experience thromboembolic complications. ${ }^{11,13}$

This study focused on AF patients on warfarin treatment and subsequent intracranial hemorrhage. We were particularly interested in determining the prevalence of traumatic and spontaneous hemorrhages and whether there are any differences in the clinical characteristics and mortality of these 2 types of intracranial bleeds.

\section{Methods}

\section{Study population}

The FibStroke registry included all consecutive patients with a previously diagnosed AF (paroxysmal, persistent, or permanent) who had an ischemic stroke or intracranial hemorrhage during the study period 2003-2012 in 2 university hospitals and 2 central hospitals (except 2006-2012 in one central hospital) in Finland. Initial screening was conducted from the hospital discharge reports with the following criteria: the patient had been diagnosed with (1) AF or atrial flutter and (2) stroke, transient ischemic attack (TIA), or intracranial hemorrhage. In this substudy, we included all intracranial hemorrhage patients who had been previously been diagnosed with AF/atrial flutter and had warfarin treatment. A comprehensive list of the ICD-10 codes used for the screening is provided in the e-Methods (links.lww.com/CPJ/A39). After the initial screening, all patient files were reviewed individually and the diagnoses of AF or atrial flutter and the diagnoses of stroke, TIA, or intracranial hemorrhage were confirmed case by case. For each case, patient characteristics, risk factors for ischemic stroke and hemorrhage, medication, laboratory results, major operations and bleeding events during the 30 days preceding the stroke, TIA, or intracranial hemorrhage were recorded and stored in a structured electronic case report form.

Intracranial hemorrhages were classified according to its localization into intracerebral hemorrhages ( $\mathrm{ICH}$ ), subdural hemorrhages (SDH), subarachnoid hemorrhages, and combined hemorrhage, if imaging revealed the presence of hemorrhage in more than one anatomical location. In addition, the bleedings were divided into traumatic $(\mathrm{n}=234)$ and spontaneous $(\mathrm{n}=358)$ hemorrhages (table). In addition, 30-day mortality data were obtained. Data were collected in a structured electronic case report form.

\section{Standard protocol approvals, registrations, and patient consents}

The study protocol was approved by the Medical Ethics Committee of the Hospital District of Southwest Finland and the ethics committee of the National Institute for Health and Welfare. Informed consent was not required because of the registry nature of the study. The study conforms to the Declaration of Helsinki. The FibStroke trial has been registered in clinicaltrials.gov (NCT02146040).

\section{Definitions}

The diagnosis of AF was confirmed using a 12-lead ECG according to the standard criteria. All patients had had at least one confirmed AF episode prior to the intracranial hemorrhage. The diagnoses of intracranial hemorrhages were confirmed from the patient records, as diagnosed by the treating physician. An intracranial hemorrhage was considered traumatic if the bleeding was preceded by a head injury or a fall. In addition, if MRI or CT revealed intracranial hemorrhage with cerebral contusion, it was classified as a traumatic ICH. Only events assessed as definite by the treating physician were included in the study. All patients were imaged by CT or MRI. Anemia was considered as a hemoglobin level $<100 \mathrm{~g} / \mathrm{L}$ and hypertension, hypercholesterolemia, and diabetes when medication for these diseases had been prescribed prior to admission. Estimated glomerular filtration rate was calculated by MDRD formula. Modified HAS-BLED was calculated without labile international normalized ratio (INR).

\section{Statistical analysis}

Continuous variables were reported as the mean \pm SD if normally distributed, and as median (interquartile range) if they were skewed. Categorical variables were described with absolute and relative (percentage) frequencies. Comparisons between study subgroups were conducted with the MannWhitney $U$ test for continuous variables and the $\chi^{2}$ test for categorical variables. In addition, multivariable logistic regression was applied to evaluate the risk factors for 30-day mortality. Variables with $p<0.05$ between the group comparisons were entered into the model as covariates. All tests were 2 -sided, and statistical significance was set at $5 \%$. Statistical analysis was performed using IBM (Armonk, NY) SPSS Statistics software version 24.0.

\section{Data availability}

Access to study data is regulated by Finnish law. Data are available from the Turku University Hospital Institutional Data Access/Ethics Committee for researchers who meet the criteria as required by the Finnish law for access to confidential data. 
Table Demographic characteristics

\begin{tabular}{|c|c|c|c|}
\hline & Traumatic, $\mathrm{n}=234$ & Spontaneous, $\mathrm{n}=358$ & $p$ Value \\
\hline Age, y & $81(74-86)$ & $78(72-84)$ & 0.01 \\
\hline $65-75$ & $98(27)$ & $98(27)$ & 0.10 \\
\hline$>75$ & $172(74)$ & $227(63)$ & 0.01 \\
\hline Female & $96(41)$ & $150(42)$ & 0.78 \\
\hline \multicolumn{4}{|l|}{ Treatment for } \\
\hline Hypertension & $162(69)$ & $243(68)$ & 0.77 \\
\hline Diabetes & $54(23)$ & $82(23)$ & 0.96 \\
\hline Hypercholesterolemia & $92(40)$ & $118(33)$ & 0.12 \\
\hline Vascular disease & $95(41)$ & $148(41)$ & 0.86 \\
\hline Previous MI & $41(18)$ & $59(17)$ & 0.74 \\
\hline Coronary artery disease & $83(36)$ & $131(37)$ & 0.79 \\
\hline Other vascular disease & $21(9)$ & $19(5)$ & 0.08 \\
\hline Previous ischemic CE & $58(25)$ & $97(27)$ & 0.55 \\
\hline Congestive heart failure & $70(30)$ & $58(16)$ & $<0.01$ \\
\hline Permanent AF & $173(74)$ & $248(69)$ & 0.63 \\
\hline Bleeding history & $22(9)$ & $22(6)$ & 0.14 \\
\hline Anemia $(\mathrm{Hb}<100 \mathrm{~g} / \mathrm{L})$ & $15(7)$ & $10(3)$ & 0.03 \\
\hline Hemoglobin, g/L & $127(20)$ & $136(18)$ & $<0.01$ \\
\hline Alcohol abuse & $8(4)$ & $17(5)$ & 0.47 \\
\hline $\mathrm{CHA}_{2} \mathrm{DS}_{2}$-VASc score & $4(3-5)$ & $4(3-5)$ & 0.08 \\
\hline$\geq 2$ & $222(95)$ & $339(95)$ & 0.92 \\
\hline Modified HAS-BLED score & $2(2-3)$ & $2(2-3)$ & 0.05 \\
\hline$\geq 3$ & $98(42)$ & $130(36)$ & 0.17 \\
\hline eGFR, mL/min/1.73 $\mathrm{m}^{2}$ & $74(32)$ & $75(37)$ & 0.53 \\
\hline$<60$ & $74(32)$ & $109(30)$ & 0.76 \\
\hline$<30$ & $19(8)$ & $17(5)$ & 0.09 \\
\hline INR on admission & $2.7(1.3)^{a}$ & $2.7(1.1)^{b}$ & 0.79 \\
\hline $2.0-3.0$ & $110(47)$ & $179(50)$ & 0.34 \\
\hline$<2.0$ & $38(16)$ & $57(16)$ & 0.37 \\
\hline$>3.0$ & $76(33)$ & $113(32)$ & 0.34 \\
\hline Aspirin & $35(15)$ & $59(17)$ & 0.64 \\
\hline Clopidogrel & $4(2)$ & $7(2)$ & 1.00 \\
\hline NSAID & $4(2)$ & $5(1)$ & 0.74 \\
\hline SSRI & $13(6)$ & $18(5)$ & 0.85 \\
\hline $\begin{array}{l}\text { Abbreviations: } \mathrm{AF}=\text { atrial fibri } \\
\mathrm{MI}=\text { myocardial infarction; } \mathrm{N} \\
\text { The values denote mean } \pm \mathrm{S} \\
\text { a Data missing in } 10 \text { patients. } \\
\text { b } \text { Data missing in } 9 \text { patients. }\end{array}$ & $\begin{array}{l}\text { ent; eGFR = estimated } \\
\text { i-inflammatory agent } \\
\text { ). }\end{array}$ & $\begin{array}{l}\mathrm{te} ; \mathrm{Hb}=\text { hemoglobin; INR } \\
\text { nin receptor inhibitor; } \mathrm{TI}\end{array}$ & $\begin{array}{l}\text { zed ratio; } \\
\text { attack. }\end{array}$ \\
\hline
\end{tabular}


One of the main findings in our large real-life cohort of warfarin-treated patients with AF is that almost half (40\%) of intracranial hemorrhages were traumatic.

\section{Results}

The study included a total of 592 patients with intracranial hemorrhages. Of those, 234 (40\%) were traumatic and 358 (60\%) spontaneous. The clinical characteristics of the patients are depicted in the table. Patients with a traumatic hemorrhage were older ( 81 vs 78 years, $p=0.01$ ), presented more often with a history of congestive heart failure (30\% vs $16 \%, p<0.01)$, and had a higher prevalence of anemia during admission $(\mathrm{Hb}<100 \mathrm{~g} / \mathrm{L}, 7 \%$ vs $3 \%, p=0.03)$ than patients with a spontaneous hemorrhage, but otherwise there were no differences in the clinical characteristics between the groups.

Median $\mathrm{CHA}_{2} \mathrm{DS}_{2}$-VASc was 4 and median HAS-BLED 2 in both groups. The median INR on admission was 2.7 (range 1.2-13) in patients with traumatic hemorrhage and 2.7 (range $0.8-15$ ) in patients with a spontaneous hemorrhage $(p=0.79)$. INR was $>3$ in $33 \%$ of patients with a traumatic hemorrhage compared to $32 \%$ in patients with a spontaneous hemorrhage; i.e., no difference between the groups $(p=0.34)$. The majority $(64 \%)$ of the traumatic hemorrhages were SDHs, whereas most (67\%) of the spontaneous hemorrhages were ICHs (figure 1). Ninety-three (39\%) of the ICHs were lobar and 142 (59\%) were deep.

The 30-day mortality was higher in patients with spontaneous hemorrhage compared to patients with traumatic hemorrhage ( $36 \%$ vs $25 \%$, odds ratio [OR] $1.74,95 \%$ confidence interval [CI] 1.19-2.53, $p<0.01)$. The difference remained in a multivariable logistic regression analysis, including anemia and $\mathrm{CHA}_{2} \mathrm{DS}_{2}$-VASc score as covariates (OR 1.84 [95\% CI 1.25-2.72], $p=0.002$ ). In addition, as illustrated in figure 2 , mortality was higher among those with spontaneous haemorrhage irrespectively of the bleeding site.

\section{Discussion}

One of the main findings in our large real-life cohort of warfarintreated patients with $\mathrm{AF}$ is that almost half (40\%) of intracranial hemorrhages were traumatic. Traumatic hemorrhages were most often SDHs (64\%), followed by ICHs (20\%), whereas spontaneous intracranial hemorrhages were most often ICHs. Though patients with traumatic hemorrhage had lower 30-day mortality (25\%) than those experiencing a spontaneous hemorrhage (36\%), nonetheless they constitute a remarkable cause of anticoagulation-related deaths. Patients with traumatic intracranial hemorrhage were older and more often had congestive heart failure and anemia. INR values were within the therapeutic range in half of the patients at the onset of bleeding in both groups. Unexpectedly, the patients with either traumatic or spontaneous intracranial hemorrhage were similar in terms of $\mathrm{CHA}_{2} \mathrm{DS}_{2}$-VASc and HAS-BLED scores and admission INR values.

\section{Types of intracranial hemorrhages}

In our study, $40 \%$ of warfarin-related intracranial hemorrhages were of traumatic origin. Similarly to our findings, in the ARISTOTLE trial, ${ }^{14}$ the proportion of traumatic intracranial hemorrhage was $36 \%$. The high prevalence of traumatic bleeds underlines that in a patient on warfarin therapy presenting with intracranial hemorrhage, the possibility of a head trauma should always be taken into consideration irrespectively of the INR. In the RE-LY ${ }^{2}$ and ROCKET-AF ${ }^{15}$ trials, which unlike our study and the ARISTOTLE trial reported only ICHs, the corresponding percentages of traumatic origin were $11 \%$ and $7 \%$, respectively. The origin of the bleeding associates with the type of intracranial hemorrhage; most of the traumatic intracranial hemorrhages were SDHs (64\% in our study) whereas most of the spontaneous hemorrhages were ICHs (67\% in our study), which is in line with earlier reports. ${ }^{2,14}$

\section{Assessment of stroke and bleeding risk}

Advanced age increases the risk of intracerebral hemorrhage, both spontaneous and traumatic, in $\mathrm{AF}$ patients on OAC.,16,17 In our study, patients with traumatic intracerebral hemorrhage were older compared to patients

Figure 1 Types (sites) of intracranial hemorrhage

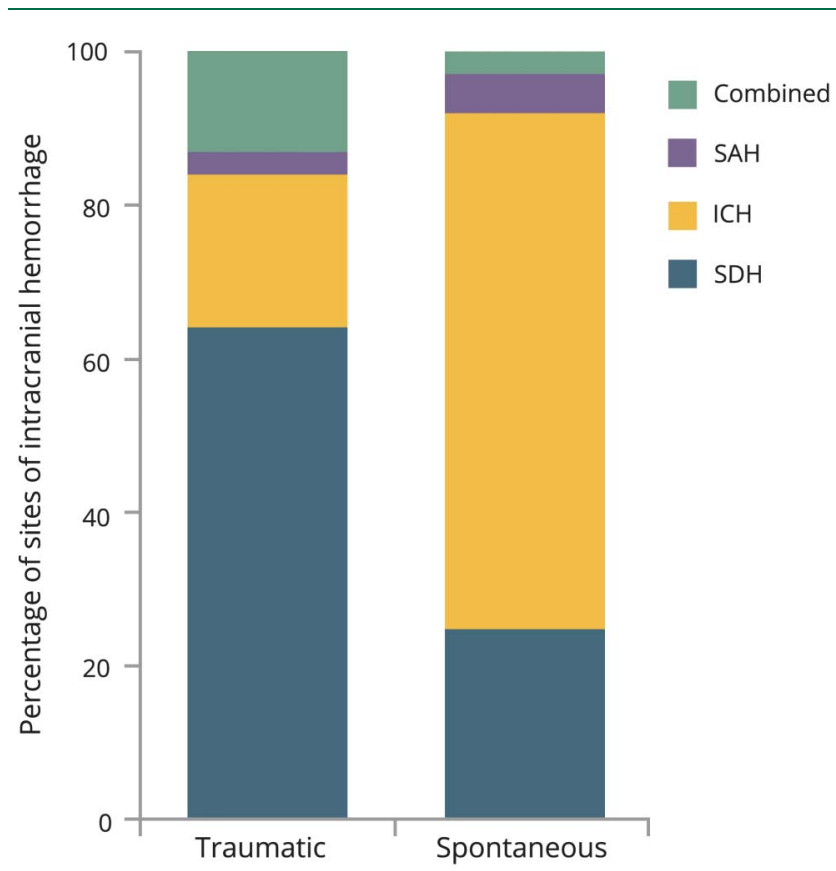

Combined indicates intracranial hemorrhage in more than one anatomical location. ICH = intracerebral hemorrhage; SAH = subarachnoid hemorrhage; $\mathrm{SDH}=$ subdural hemorrhage. 
An important issue in treating patients with AF is to find the optimal balance between the risks of bleeding and thromboembolic complications.

with spontaneous hemorrhage. Old age, age-related reduction in muscle power, and frailty predispose to falls. In addition, balance problems related to inner ear pathology $^{18}$ and orthostatic hypotension ${ }^{19}$ are age-dependent. We found also that patients with traumatic hemorrhage more often had congestive heart failure and anemia $(\mathrm{Hb}$ $<100 \mathrm{~g} / \mathrm{L}$ ) than patients with spontaneous hemorrhage. Heart failure per se is associated with increased risk of falls, ${ }^{20}$ and its treatment may be complicated by orthostatic hypotension. ${ }^{19}$ Accordingly, a link between even mild anemia and the risk of falls in elderly patients has been reported. ${ }^{21}$

An important issue in treating patients with $\mathrm{AF}$ is to find the optimal balance between the risks of bleeding and thromboembolic complications. HAS-BLED is most commonly used to assess the risks of bleeding associated with oral anticoagulation. In addition, falls, which are not listed in the
HAS-BLED score, increase the risk of ICH. Considering the high proportion (40\%) of traumatic bleedings in our study, our results suggests that in AF patients the risk of falls should be included in the risk evaluation of bleeding, and other options for stroke prevention, such as left atrial appendage closure, should be considered in patients with a high risk of falls.

Another real-life challenge is that the risks of stroke and bleeding often overlap. Patients with the highest risk of stroke also possess a risk of bleeding. This was true also in our study. There were no differences in the median CHA2DS2-VASc and HAS-BLED scores between the groups. In addition, $\mathrm{CHA}_{2} \mathrm{DS}_{2}$-VASc was higher than HAS-BLED in patients with traumatic and spontaneous bleeding. These results are in line with Hart et al. ${ }^{2}$ They reported that risk factors, including CHADS2 score, for subdural hemorrhages were similar in patients with spontaneous and traumatic SDHs. Our results and those of Hart et al. ${ }^{2}$ also suggest that despite the risk of bleeding, most patients benefit from anticoagulation and starting and withholding OAC should not be based solely on the evaluation of the risk of stroke $\left(\mathrm{CHA}_{2} \mathrm{DS}_{2}\right.$-VASc) or bleeding (HAS-BLED) but a more broader evaluation of risks.

The combination of OAC and antiplatelet therapy, selective serotonin reuptake inhibitors (SSRIs), or nonsteroidal anti-

Figure 2 Thirty-day mortality according to the type (site) of intracranial hemorrhage

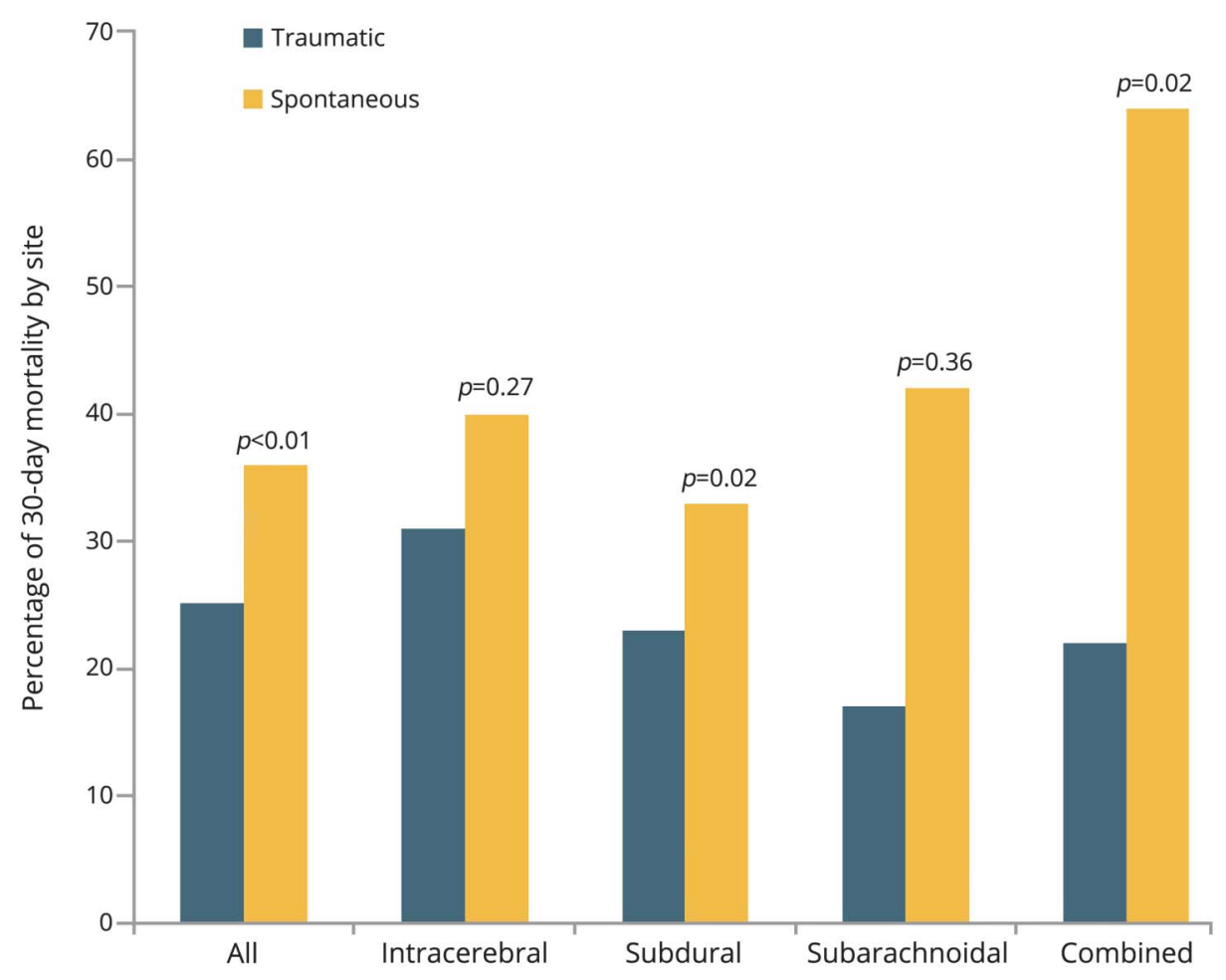

Values denote $\mathrm{n}(\%)$. Combined includes intracranial hemorrhages in more than one anatomical location. Data missing in 22 patients. 
inflammatory drugs (NSAIDs) is known to increase the risk of bleeding. ${ }^{12,16}$ In our study, patients with traumatic and spontaneous hemorrhages did not differ with respect to the simultaneous use of antiplatelet therapy, SSRIs, or NSAIDs. The combination of antiplatelet therapy and anticoagulation was relatively rare $(17 \%-19 \%)$ in our patient population.

\section{Admission INR}

High INR (especially INR >4.0) increases the risk of intracranial hemorrhage. ${ }^{8,22,23}$ In line with several previous studies, half of the intracranial hemorrhages in our study occurred with admission INR within the therapeutic range and $67 \%-68 \%$ with INR $\leq 3.0$. Hence, clinically important conclusions are that it is important to consider the possibility of intracranial hemorrhage in all anticoagulated patients with head trauma irrespective of INR and INR cannot be used to differentiate spontaneous from traumatic bleeding. Our results are in line with the work of Lopes et al., ${ }^{14}$ who reported that $79 \%$ of intracranial hemorrhages occurred when INR was $<3.0$. The median INR values preceding the bleeding event were also similar in our study and that of Lopes et al. ${ }^{14}$ (2.7 vs 2.6$)$. Labile INR values are known to increase the risk of thromboembolic and bleeding complications. . $^{8,23,24}$ In our study, one third of patients had admission INR $>3.0$, whereas INR $<2.0$ was found in only $16 \%$ of patients in both groups, values in line with previous studies. $^{25,26}$ Unexpectedly, median INR levels were similar between patients with traumatic and spontaneous hemorrhage.

\section{Mortality}

Mortality after intracerebral hemorrhage was high (31.6\%), which is in good agreement with earlier studies reporting mortality from $32 \%$ to $43 \% .^{2,14,15,27}$ In our study, traumatic hemorrhage was less often fatal than after a spontaneous one (25\% vs $36 \%$ ), also in accordance with an earlier report by Hart et al., ${ }^{2}$ where mortality after traumatic and spontaneous hemorrhage was $21 \%$ and $41 \%$, respectively. It is also of interest that all subtypes of intracranial hemorrhages (bleeding sites) were associated with lower risk of death if they were of traumatic origin. Our study was underpowered to address the statistical significance of differences between the subgroups. This should be evaluated in larger studies in the future.

\section{Limitations}

Although the study population consists of a large group of real-life and consecutive warfarin-treated patients with intracranial hemorrhage, our study has several limitations. It is retrospective and observational. Preceding trauma energy, Glasgow Coma Scale, volume of intracranial hemorrhage, and treatment were not assessed. We could only reliably access admission INR values, although it would have been ideal to have time in therapeutic range for several months preceding the intracranial hemorrhage. Moreover, patients with intracranial hemorrhage who died before hospital admission were not included.

\section{Conclusions}

A remarkable proportion of intracranial hemorrhages in AF patients on warfarin therapy are of traumatic origin. Patients with traumatic hemorrhages are older and more often have anemia and heart failure, all factors associated with increased risk of falls, when compared to patients with spontaneous hemorrhage. While there is somewhat less mortality after traumatic hemorrhage compared to a spontaneous event, they both account for a major proportion of the morbidity and mortality. Finally, INR within target range does not exclude intracranial hemorrhage and cannot be used to differentiate between traumatic and spontaneous hemorrhages.

\section{Author contributions}

H. Lehtola: acquisition of data, analysis and interpretation, critical revision of the manuscript for important intellectual content. A. Palomäki: acquisition of data, analysis and interpretation, critical revision of the manuscript for important intellectual content. P. Mustonen: study concept and design, critical revision of the manuscript for important intellectual content, study supervision. P. Hartikainen: study concept and design, critical revision of the manuscript for important intellectual content. T. Kiviniemi: study concept and design, critical revision of the manuscript for important intellectual content. H. Sallinen: acquisition of data, analysis and interpretation. I. Nuotio: study concept and design. A. Ylitalo: study concept and design. K.E.J. Airaksinen: study concept and design, critical revision of the manuscript for important intellectual content, study supervision. J. Hartikainen: study concept and design, critical revision of the manuscript for important intellectual content, study supervision.

\section{Acknowledgment}

The authors thank Tuija Vasankari for the coordination of the data sampling.

\section{Study funding}

This study was supported by the Finnish Foundation of Cardiovascular Research and the Finnish Medical Foundation.

\section{Disclosures}

H. Lehtola receives research support from the Finnish Foundation for Cardiovascular Research. A. Palomäki has received speaker honoraria from Bayer and MSD and receives research support from the Finnish Medical Foundation. P. Mustonen serves on scientific advisory boards for Boehringer Ingelheim, Bayer, Pfizer, Roche, Bristol-Myers Squibb, and Leo Pharma; and has received speaker honoraria from Orion, Boehringer Ingelheim, Bayer, Pfizer, BristolMyers Squibb, Sanofi-Aventis, and Leo Pharma. P. Hartikainen has received speaker honoraria from Genzyme, Novartis, Biogen Idec, TEVA, and Sanofi-Aventis. T. Kiviniemi serves on scientific advisory boards for MSD and Boehringer Ingelheim; serves as Associate Editor for Sydänääni, the official journal of the Finnish Cardiac Society; 
serves on speakers' bureaus for Bayer, Boehringer Ingelheim, MSD, BMS-Pfizer, and AstraZeneca; and receives research support from Atricure Ltd., USA, Finnish Medical Foundation, and Finnish Foundation for Cardiovascular Research. H. Sallinen, I. Nuotio, and A. Ylitalo report no disclosures. K.E.J. Airaksinen has received speaker honoraria from Bayer, Cardiome, Pfizer, AstraZeneca, and Boehringer Ingelheim and receives research support from the Finnish Foundation for Cardiovascular Research. J. Hartikainen serves on scientific advisory boards for AstraZeneca, Amgen, and Bayer; has received speaker honoraria from Cardiome, St Jude Medical, and Biotronic; and receives research support from European Union Seventh Framework Program and Horizon 2020 program and the Finnish Foundation for Cardiovascular Research. Full disclosure form information provided by the authors is available with the full text of this article at Neurology.org/cp.

Received February 6, 2018. Accepted in final form April 16, 2018.

\section{References}

1. Giugliano RP, Ruff CT, Braunwald E, et al. Edoxaban versus warfarin in patients with atrial fibrillation. N Engl J Med 2013;369:2093-2104.

2. Hart RG, Diener HC, Yang S, et al. Intracranial hemorrhage in atrial fibrillation patients during anticoagulation with warfarin or dabigatran: the RE-LY trial. Stroke 2012;43:1511-1517.

3. Hylek EM, Held C, Alexander JH, et al. Major bleeding in patients with atrial fibrillation receiving apixaban or warfarin: the ARISTOTLE trial (apixaban for reduction in stroke and other thromboembolic events in atrial fibrillation): predictors, characteristics and clinical outcomes. J Am Coll Cardiol 2014;63:2141-2147.

4. Patel MR, Mahaffey KW, Garg J, et al; ROCKET AF Investigators. Rivaroxaban versus warfarin in nonvalvular atrial fibrillation. N Engl J Med 2011;365:883-891.

5. Sjögren V, Grzymala-Lubanski B, Renlund H, et al. Safety and efficacy of well managed warfarin: a report from the Swedish quality register Auricula. Thromb Haemost 2015;113:1370-1377.

6. Dowlatshahi D, Butcher KS, Asdaghi N, et al; Canadian PCC Registry (CanPro) Investigators. Poor prognosis in warfarin-associated intracranial hemorrhage despite anticoagulation reversal. Stroke 2012;43:1812-1817.

7. Gage BF, Birman-Devch E, Kerzner R, Radford MJ, Nilasena DS, Rich MW. Incidence of intracranial hemorrhage in patients with atrial fibrillation who are prone to fall. Am J Med 2005;118:612-617.

8. Fang MC, Chang Y, Hylek EM, et al. Advanced age, anticoagulation intensity, and risk for intracranial hemorrhage among patients taking warfarin for atrial fibrillation. Ann Intern Med 2004;141:745-752.
9. Bromfield SG, Ngameni CA, Colantonio LD, et al. Blood pressure, antihypertensive polypharmacy, frailty, and risk for serious fall injuries among older treated adults with hypertension. Hypertension 2017;70:259-266.

10. Pisters R, Lane DA, Nieuwlaat R, de Vos CB, Crijns HJ, Lip GY. A novel user-friendly score (HAS-BLED) to assess 1-year risk of major bleeding in patients with atrial fibrillation: the Euro Heart Survey. Chest 2010;138:1093-1100.

11. Donze J, Clair C, Hug B, et al. Risk of falls and major bleeds in patients on oral anticoagulation therapy. Am J Med 2012;125:773-778.

12. Quinn GR, Singer DE, Chang Y, et al. Effect of selective serotonin reuptake inhibitors on bleeding risk in patients with atrial fibrillation taking warfarin. Am J Cardiol 2014; 114:583-586.

13. Lip GY, Nieuwlaat R, Pisters R, Lane DA, Crijns HJ. Refining clinical risk stratification for predicting stroke and thromboembolism in atrial fibrillation using a novel risk factor-based approach: the Euro Heart Study. Chest 2010;137:263-272.

14. Lopes RD, Guimarães PO, Kolls BD, et al. Intracranial hemorrhage in patient with atrial fibrillation receiving anticoagulation therapy. Blood 2017;129:2980-2987.

15. Hankey GJ, Stevens SR, Piccini JP, et al. Intracranial hemorrhage among patients with atrial fibrillation anticoagulated with warfarin or rivaroxaban: the rivaroxaban once daily, oral, direct factor Xa inhibition compared with vitamin $\mathrm{K}$ antagonism for prevention of stroke and embolism trial in atrial fibrillation. Stroke 2014;45: 1304-1312.

16. Lip GY, Frison L, Halperin JL, Lane DA. Comparative validation of a novel risk score for predicting bleeding risk in anticoagulated patients with atrial fibrillation: the HASBLED (hypertension, abnormal renal/liver function, stroke, bleeding history or predisposition, Labile INR, elderly, drugs/alcohol concomitantly) score. J Am Coll Cardiol 2011;57:173-180.

17. Björck F, Renlund H, Lip GY, Wester P, Svensson PJ, Själander A. Outcomes in a warfarin-treated population with atrial fibrillation. JAMA Cardiol 2016;1: $172-180$

18. Parham K, Kuchel GA. A geriatric perspective on benign paroxysmal positional vertigo. J Am Geriatr Soc 2016;64:378-385.

19. Ricci F, DeCaterina R, Fedorowski A. Orthostatic hypotension: epidemiology, prognosis, and treatment. J Am Coll Cardiol 2015;66:848-860.

20. Jansen S, Bhangu J, deRooij S, Daams J, Kenny RA, van der Velde N. The association of cardiovascular disorders and falls: a systematic review. J Am Med Dir Assoc 2016; 17:193-199.

21. Dharmarajan TS, Norkus EP. Mild anemia and the risk of falls in older adults from nursing homes and the community. J Am Med Dir Assoc 2004;5:395-400.

22. McGrath ER, Kapral MK, Fang J, et al; Investigators of the Registry of the Canadian Stroke Network. Which risk factors are more associated with ischemic stroke than intracerebral hemorrhage in patients with atrial fibrillation? Stroke 2012;43: 2048-2054.

23. Hylek EM, Go AS, Chang Y, et al. Effect of intensity of oral anticoagulation on stroke severity and mortality in atrial fibrillation. N Engl J Med 2003;349:1019-1026.

24. White HD, Gruber M, Feyzi J, et al. Comparison of outcomes among patients randomized to warfarin therapy according to anticoagulant control: results from SPORTIF III and V. Arch Intern Med 2007;167:239-245.

25. Curtze S, Strbian D, Meretoja A, et al. Higher baseline international normalized ratio value correlates with higher mortality in intracerebral hemorrhage during warfarin use. Eur J Neurol 2014;21:616-622.

26. Fong MK, Sheng B, Chu YP, et al. Atrial fibrillation patients who sustained warfarinassociated intracerebral haemorrhage have poor neurological outcomes: results from a matched case series. Hong Kong Med J 2017;13:117-121.

27. Giugliano RP, Ruff CT, Wiviott SD, et al. Mortality in patients with atrial fibrillation randomized to edoxaban or warfarin: insights from the ENGAGE AF-TIMI 48 trial. Am J Med 2016;129:850-857.

\section{Share Your Insights, Expertise, and Experiences}

- How are you employing drugs and devices in your field?

- What ethical challenges do you face?

- Do you have a case report that is illustrative of a clinical challenge?

- What challenges have you faced or successes have you enjoyed in bringing greater efficiency to your practice?

Deliver a high-quality, peer-reviewed message to your colleagues in practice, submit your paper at NPub.org/NCP/submit. 